\title{
The Optimal Solution to the Development of Water Resources Influencing Factors Based on GRNN and the Genetic Algorithm Model: Case of Tianjing
}

\author{
Keke Fang
}

Department of Power Engineering, North China Electric Power University, Baoding, Hebei 071003, PR China

1098364379@qq.com

Keywords: Optimal solution; GRNN; The Genetic Algorithm.

\begin{abstract}
According to GRNN, the paper find the network of the relationship between geographical, economic and environmental factors and its influencing factors .According to the genetic algorithm, we detect four indicators influencing mainly the ability among the 9 indicators. Our plan of intervention aims to reduce the impact of these four factors on ability to develop Water Resource in the future. Finally, according to our plan of intervention, we can get the best conditions of all indicators in the future.
\end{abstract}

\section{Introduction}

Nonlinear systems exist in many areas, and the optimal solution to nonlinear systems needs to be solved to determine the future direction of the field, but often do not know the system function between the input and output expressions. Therefore, neural network can be used to solve, and then use genetic algorithm to solve the optimal solution.An example as the system of influencing factors of sustainable development of water resources, in order to get the optimal solution, first using GRNN got the network of the relationship between geographical, economic and environmental factors and its influencing factors, and then using genetic algorithm found out the reasonable range of each index Take the optimal solution of each factor.

\section{The Development of Water Resources Influencing Factors Model}

\subsection{The Establishment of GRNN Model}

\subsubsection{The Analysis of GRNN Model}

The GRNN neural network consists of four layers, including the input layer, the model layer, the summation layer and the output layer. We establish four GRNN neural networks for the four principle layer respectively[1]. As is shown on Figure 1.

Input layer Model layer The summation layer Output layer

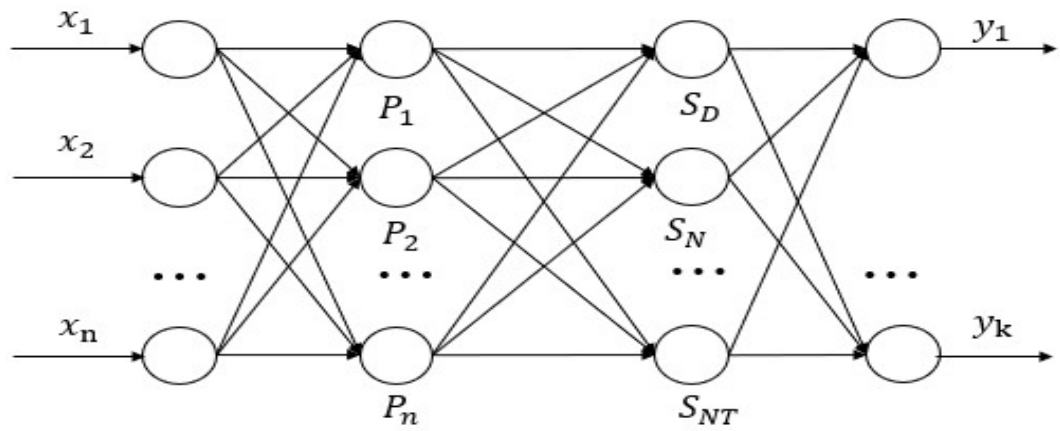

Figure 1. The schematic diagram of GRNN.

The input layer

The number of input neurons is equal to the input vector dimension in the learning sample. Each neuron is a simple distribution unit that passes input variables directly to the mode layer 
The Model layer

The number of neuron in the model layer is the number of learning samples. Each neuron corresponds to its own sample, which is the transfer function of neuron in this layer can be represented as:

$$
p_{i}=\exp \left[-\frac{\left(X-X_{i}\right)^{T}\left(X-X_{i}\right)}{2 \sigma^{2}}\right] \quad i=1,2, \cdots 5
$$

Where $\mathrm{x}$ is the input variables in the network, ${ }^{X_{i}}$ is the learning sample of the ${ }^{i}$ Th neuron. $\sigma_{\text {is }}$ the smoothing factor

The summation layer

This layer calculates the sum of two types of neurons using arithmetic summation and weighted summation respectively.

The result of arithmetic summation $\left(S_{D}\right)$ is the sum of the output of all neurons $\left(P_{i}\right)$ in the model layer. The formula can be shown as:

$$
S_{D}=\sum_{i=1}^{4} P_{i}
$$

The result of weighted summation $\left(S_{N j}\right)$ is the sum of all products of the output of all neurons $\left(P_{i}\right)$ in the model layer and its corresponding weights $\left(y_{i j}\right)$

The output layer

$$
S_{N j}=\sum y_{i j} p_{i} \quad j=1
$$

The output of the network is the level of the economic factors. The number of neurons in the output layer is the dimension of the output vector in the learning sample. The output neuron is our evaluating result which can be shown as:

$$
Y=\frac{S_{N j}}{S_{D}} \quad j=1
$$

\subsection{The Establishment of Neural Network and Genetic Algorithm Model}

The genetic algorithm is a genetic algorithm that selects, crosses and mutates to produce the next population in the course of natural evolution. By using this algorithm, we can find the minimum value of the dependent variable in the range of the independent variable, so as to realize the optimal value [2]

\subsubsection{The Analysis of the Genetic Algorithm Model}

Genetic algorithm individual coding

First of all, we randomly select Pop_Size individuals within a reasonable range. The ith individual of the genetic algorithm is:

$$
X_{i}=\left(x_{i 1}, x_{i 2}, \ldots, x_{\text {in }}\right) \quad i=1,2, \cdots, \text { Pop_Size }
$$

Where the gene value $x_{i 1}, x_{i 2}, \ldots, x_{i n}$ is a random number over the range of independent variables, $\mathrm{Xi}$ is the chromosome of the individual, and Pop_Size is the population size.

Then, the individual is binary coded as an-tuple vector, that is: $X_{i}=\left(x_{1}, x_{2}, \cdots, x_{n}\right)$

Calculation of individual fitness values

For each individual, the gene values are entered into the trained neural network to obtain the output value. In order to reflect the adaptability of staining, the fitness function is introduced $f\left(X_{i}\right)$

Genetic selection

$$
f\left(X_{i}\right)=\operatorname{sim}\left(\text { net }, X_{i}\right)
$$

Selection is based on the evaluation of fitness values. Let

$$
f_{1}\left(X_{k}\right)=1 / f\left(X_{k}\right)
$$

Calculate the survival probability of every individual: 


$$
p_{i}=f_{1}\left(X_{i}\right) / \sum_{i=1}^{\text {Pop_Size }} f_{1}\left(X_{i}\right)
$$

Use Roulette to choose the individuals with large survival probability, that is, individuals with small fitness values.

Genetic crossover

Two individuals are randomly selected to carry out the crossover operation with the crossover probability, and the crossover method adopts the linear crossover. We assume that the crossover probability is 0.4 . For example, when a linear combination of individuals $X_{i}=\left(x_{i 1}, x_{i 2}, \cdots, x_{i n}\right)$ and $X_{j}=\left(x_{j 1}, x_{j 2}, \cdots, x_{j n}\right)$ is crossed, two new individuals $X_{i}^{\prime}=\lambda X_{i}+(1-\lambda) X_{j}$ and $X_{j}^{\prime}=(1-\lambda) X_{i}+\lambda X_{j}$ are generated, where the linear combination coefficient $\lambda$ is a constant between 0 and 1 .

Genetic mutation

Select a gene in an individual with mutation probability $p_{m}$, generate a random number in the solution space and replace the selected gene with this random number.

During the genetic manipulation of each generation, the best individual of the generation is

saved to speed up the rate of convergence. If the maximum algebra has been reached, the genetic operation is ended, otherwise the selection continues.

\subsection{Preparatory Work for Data}

Firstly, we normalize all data. According to the relevant references, we adopt the standardize deviation method to standardize all data and map them into the interval from 0 to 1 . The result of normalize $\mathrm{x}$ be assumed as $\mathrm{x}^{*}$, whose equation can be represented as:

$$
x^{*}=\frac{x-\mathrm{min}}{\mathrm{max}-\mathrm{min}}
$$

Where max represents the maximum of sampled data and min represents the minimum of sampled data.

\subsection{The Training and Test of GRNN Network}

In this paper, GRNN assessed the impact of regional water resources, shown in Figure 1. Taking the economic factors X3, X4, X5 and A2 in Table 1 as an example, each evaluation index in A2 is used as an input vector, that is, the number of neurons in the input layer is 3 . The interpolation result of 1 to 5 is used as the output vector, that is, the output. The number of neurons in a layer is 1 . This paper uses interpolation to select 100 samples for training

\begin{tabular}{|c|c|c|c|c|c|c|c|}
\hline \multirow{2}{*}{ factors } & \multirow{2}{*}{ Indicator } & \multicolumn{6}{|c|}{ The specific standard values corresponding to different level } \\
\hline & & 1 & 2 & 3 & 4 & 5 & range \\
\hline \multirow{2}{*}{ The geographical factors(A1) } & The amount of precipitation $(\mathrm{X} 1)$ & $\geq 1500$ & $\geq 1200$ & $\geq 1000$ & $\geq 800$ & $<800$ & $400 \sim 3000$ \\
\hline & The proportion of surface water and groundwater(X2) & $\geq 80$ & $\geq 60$ & $\geq 40$ & $\geq 20$ & $<20$ & $10 \sim 160$ \\
\hline \multirow{3}{*}{ The economic factors(A2) } & The water demand quota of agricultural irrigation(X3) & $\leq 30$ & $\leq 50$ & $\leq 100$ & $\leq 200$ & $>200$ & $10 \sim 300$ \\
\hline & The water demand of a unit industrial added value(X4) & $\leq 3000$ & $\leq 4500$ & $\leq 6000$ & $\leq 7500$ & $>7500$ & $1500 \sim 10000$ \\
\hline & The proportion of recycled water(X5) & $\leq 10$ & $\leq 40$ & $\leq 55$ & $\leq 70$ & $>70$ & $5 \sim 100$ \\
\hline \multirow{4}{*}{ The environmental factors(A3) } & The per capita water resources(X6) & $\geq 90$ & $\geq 80$ & $\geq 40$ & $\geq 20$ & $<20$ & $0 \sim 100$ \\
\hline & The water demand of a unit GDP(X7) & $\leq 10$ & $\leq 15$ & $\leq 25$ & $\leq 30$ & $>30$ & $6 \sim 100$ \\
\hline & $\begin{array}{l}\text { The proportion of water demand for environmental } \\
\text { protection(X8) }\end{array}$ & $\leq 5$ & $\leq 10$ & $\leq 20$ & $\leq 25$ & $>25$ & $1 \sim 30$ \\
\hline & The sewage produced of a unit GDP(X9) & $\geq 90$ & $\geq 75$ & $\geq 60$ & $\geq 40$ & $<40$ & $20 \sim 100$ \\
\hline
\end{tabular}

Table 1 . The different level and its corresponding specific standard values 


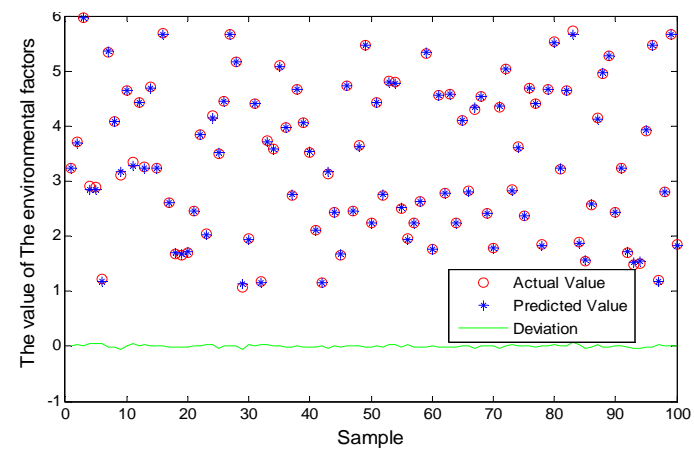

Figure 2. The test of the network's training

As is shown by the results, the data resulting from network's training is great when the value of network's training is 0.1 . The smaller the value of learning speed, the smaller the deviation between samples and network is. As is shown on Figure2, GRNN training to get better results.

\subsection{Implantation of Neural Network Genetic Algorithm}

Taking Tianjing as an example, in the range allowed by each index of Tianjin, Municipality, the algorithm is solved as shown in Table 2

Table 2. The optimal solution of each index

\begin{tabular}{cccccccccc} 
Indicator & $\mathrm{X} 1$ & $\mathrm{X} 2$ & $\mathrm{X} 3$ & $\mathrm{X} 4$ & $\mathrm{X} 5$ & $\mathrm{X} 6$ & $\mathrm{X} 7$ & $\mathrm{X} 8$ & $\mathrm{X} 9$ \\
value & 528 & 43.433 & 11 & 1608.784 & 5.025 & 11.225 & 40.117 & 1.049 & 46.967 \\
\hline
\end{tabular}

\section{Conclusion}

For Tianjing, the economic factor can be greatly enhanced, and the economic impact factors in water resources can be emphasized. Using neural network genetic algorithm, we can get the optimal solution without knowing the dependent variable and dependent variable. The algorithm is widely used and can be widely promoted.

\section{References}

[1]. CUI Dongwen, " Evaluation of sustainable utilization of regional water resources based on GRNN neural network model: case of Wenshan Prefecture of Yunnan Province," Yangtze River, Vol.43, no.5, pp.26-31, Mar. 2012

[2]. XU Xin-ai, "Optimal Solution to Nonlinear System Based on the Neural Network Genetic Algorithm," Jiangxi Institute of Education, vol.34, no.03, pp.20-23, 2013 